USC-93-020

\title{
Lattice regularization of massive and massless integrable field theories
}

\author{
N.Yu Reshetikhin ${ }^{\dagger}$, H.Saleurl \\ $\dagger$ Department of Mathematics, Berkeley University \\ Berkeley CA 94720 \\ - Department of Physics and Department of Mathematics \\ University of southern California \\ Los Angeles CA 90089
}

\begin{abstract}
We show that integrable vertex and RSOS models with trigonometric Boltzmann weights and appropriate inhomogeneities provide a convenient lattice regularization for massive field theories and for the recently studied massless field theories that interpolate between two non trivial conformal field theories. Massive and massless S matrices are computed from the lattice Bethe ansatz.
\end{abstract}

\section{Introduction}

The perturbation of unitary minimal models $\mathcal{M}_{t}$ of conformal field theory with central charge

$$
c=1-\frac{6}{t(t-1)},
$$

by the $\phi_{13}$ operator of conformal weight

$$
h=\frac{t-2}{t},
$$

\footnotetext{
1 Packard Fellow
} 
is known to give an integrable $1+1$ quantum field theory [1]: $\mathcal{M} A_{t}^{ \pm}$. The infra red (IR) properties of this theory depend on the sign of the coupling. Writing the hamiltonian as

$$
H_{\mathcal{M} A_{t}}=H_{\mathcal{M}_{t}}+g \int \phi_{13}(x) \bar{\phi}_{13}(x) d x
$$

the case $g<0$ gives the massive integrable field theory $\mathcal{M} \mathcal{A}_{t}^{-}$with a trivial IR fixed point while the case $g>0$ is "massless" and gives $\mathcal{M A}_{t}^{+}$with an IR fixed point that coincides with $\mathcal{M}_{t-1}$ [1]] [2].

The conformal field theory $\mathcal{M}_{t}$ is well known to describe the continuum limit of the $(t-2)^{t h}$ critical Ising model [3], a critical point where in the Landau Ginzburg picture $t-2$ minima of the potential coalesce. The massive flow describes the flow towards a phase where these minima separate and are degenerate and where spontaneous symmetry breaking occurs, while the massless one corresponds to one minima decoupling so in the IR the model is a multicritical Ising model with one order of criticality less.

It is known that minimal models $\mathcal{M}_{t}$ of conformal field theory can be obtained by twisting of boundary conditions and then reduction of the Gaussian model with $c=1$ [4]. In a similar way, the massive regime of the perturbed theory with action (1.3) can be described as a reduction of the sine-Gordon model [5] at coupling $\frac{\beta^{2}}{8 \pi}=\frac{t-1}{t}$. Due to twisting, the coupling constant in front of the cosine term scales as $G \propto(-g)^{1 / 2}$. The soliton mass $m$ scales as $m \propto(-g)^{\frac{t}{4}}$.

Formally the same correspondence between the two theories should also hold in the massless regime. In that case however the sine-Gordon coupling constant $G$ turns out to be purely imaginary due to the above scaling. This is a regime that has not been much studied so far, and which presents subtleties [6]. Notice of course that the rather violent non unitarity of this sine-Gordon model does not prevent the subset of observables corresponding to the minimal model to be unitary.

Perturbations of coset models $s u(2)_{s} \otimes s u(2)_{t-s-2} / s u(2)_{t-2}(s=1$ above) by the operator of dimension $h=\frac{t-2}{t}$ also give integrable models. We will denote them $\mathcal{M} A_{s, t}^{ \pm}$ depending on the sign of the coupling constant in front of the perturbation. These models can also be obtained by reduction of some other integrable models: the higher spin or fractional supersymmetric generalizations of the sine-Gordon model [7] [8].

Further generalizations to coset models of type $G_{k} \times G_{l} / G_{k+l}$ are also possible. For each such model there is a natural integrable direction of deformation [9] with the perturbing field of dimension $1-\frac{h^{v}}{k+l+h^{v}}$ (where $h^{v}$ is the dual Coxeter number) leading to 
two different theories depending on the sign of the coupling constant. The role of the sine-Gordon model is then played by its Toda generalizations and further fractional supersymmetric generalizations [10].

We show in this paper how inhomogeneous vertex or RSOS models with trigonometric Boltzmann weights and appropriate inhomogeneities provide, in their scaling limit, a general lattice regularization of the above massive and massless field theories. This allows us in particular to put the massless scattering of [11] on firmer grounds, and to prove a conjecture in [6]. In a way our approach is parallel to the one developed on [12] for non linear sigma models. It is interesting that the lattice version of the sine-Gordon model which was studied in [13] turns out to be of the same nature. It was shown in [14] that it can also be formulated as a model on the inhomogeneous lattice where at each vertex there is a cyclic representation of $U_{q} s l(2)$.

Although the method is general, we discuss mainly the $s u(2)$ case. Appropriate extensions to other groups are discussed in the last section. In section 2 we recall necessary results about the Bethe ansatz for inhomogeneous vertex and RSOS models. In section 3 we discuss the thermodynamic limit. In section 4 we consider the scaling limit. In section 5 we study the $\mathrm{S}$ matrix in the massless case and prove the conjecture of [6] . In section 6 we discuss some examples of the method applied to other groups.

Lattice regularizations of masssive field theories which are based on inhomogeneous integrable models with trigonometric Boltzmann weights have some advantages compared with the elliptic ones [15]. One is that in this case the lattice and continuum theory have the same symmetry. Another is that we know far more about the spectrum of trigonometric transfer matrices than about the elliptic ones. Finally for Lie algebras other than $s u(n)$ we do not expect elliptic vertex models [16].

\section{Inhomogeneous lattice models of $X X Z$ type}

\subsection{Vertex models}

Consider a one dimensional lattice with $\mathrm{N}$ vertices formed by the intersection of $\mathrm{N}$ columns and one row. We assume the vertices are numbered from left to right. A $U_{q} \operatorname{sl}(2)$ or generalized 6 -vertex model on such lattice can be described as follows. Fix spins $s_{j}$, $j=1, \ldots, N$ (in units where the fundamental has spin one) and an additional spin $s$. With

each vertical edge we associate the space of states $C^{s_{j}+1}$ and a complex number $u_{j}$. With each horizontal edge we associate $C^{s+1}$ and a complex number $u$. 


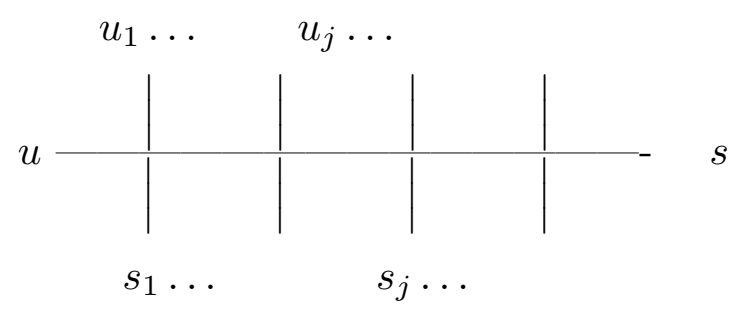

With each vertex we associate a system of Boltzmann weights which are given by $\mathrm{R}$ matrices acting in the tensor product of corresponding spaces. In each space $C^{s+1}$ we enumerate the basis by $a=1, \ldots, s+1$. Fix an element of the basis in each space of states corresponding to the boundary edges, say $a, a_{1}, \ldots, a_{N}, b, b_{1}, \ldots, b_{N}$. The partition function of the $U_{q} s l(2)$ model with the parameters and boundary conditions just described has the following form

$$
T^{s}\left(u\left|\left\{u_{j}\right\}\right|\left\{s_{j}\right\}\right)_{b, b_{1} \ldots b_{N}}^{a, a_{1} \ldots a_{N}}=\sum_{c_{1}, \ldots, c_{N-1}} R^{s s_{1}}\left(u-u_{1}\right)_{c_{1} b_{1}}^{a a_{1}} \ldots R^{s s_{N}}\left(u-u_{N}\right)_{b b_{N}}^{c_{N-1} a_{N}}
$$

Here $R^{s s_{j}}$ is the $U_{q} s l(2)$ R-matrix described in [17] and [18].

From this partition function with open boundary conditions on one row we can construct corresponding partition functions on rectangular lattices with various boundary conditions as traces of products of (2.1) .

Since the R-matrices satisfy the Yang Baxter equation we have commutativity of traces

$$
\left[t^{s}\left(u\left|\left\{u_{j}\right\}\right|\left\{s_{j}\right\}\right), t^{s^{\prime}}\left(v\left|\left\{u_{j}\right\}\right|\left\{s_{j}\right\}\right)\right]=0 .
$$

Here the transfer matrices $t^{s}\left(u\left|\left\{u_{j}\right\}\right|\left\{s_{j}\right\}\right)$ act in the tensor product $\mathcal{H}_{s_{1}, \ldots, s_{N}}^{v e r t e x}=C^{s_{1}+1} \otimes$ $\ldots \otimes C^{s_{N}+1}$ and they are given by traces of (2.1) with respect to "horizontal spaces"

$$
t^{s}\left(u\left|\left\{u_{j}\right\}\right|\left\{s_{j}\right\}\right)_{b_{1} \ldots b_{N}}^{a_{1} \ldots a_{N}}=\sum_{a=1}^{s+1} d_{a} T^{s}\left(u\left|\left\{u_{j}\right\}\right|\left\{s_{j}\right\}\right)_{a, b_{1} \ldots b_{N}}^{a, a_{1} \ldots a_{N}}
$$

where $d_{a}=\exp [\kappa(a-1-s / 2)]$ for some complex number $\kappa$. These transfer matrices commute with the total third component $s^{z}$ of the spin. The space $\mathcal{H}_{s_{1}, \ldots, s_{N}}^{v e r t e x}$ we may call vertical space. We can thus regard (2.2) as a generating function for a family of quantum integrable systems. The commuting family can be diagonalized simultaneously. The eigenvectors of $t^{s}\left(u\left|\left\{u_{j}\right\}\right|\left\{s_{j}\right\}\right)$ are parametrized by solutions $\left\{\alpha_{k}\right\}$ to the Bethe equations

$$
\prod_{j=1}^{j=N} \exp \left[i p_{s_{j}}\left(\alpha_{k}-2 i u_{j}\right)\right] \prod_{l=1}^{l=M} \exp \left[i \phi\left(\alpha_{k}-\alpha_{l}\right)\right]=-1,
$$


where $M=\left(\sum_{j} s_{j}-s^{z}\right) / 2$ is an integer. The functions $p_{s}$ and $\phi$ are given respectively by

$$
\exp i p_{s}(\alpha)=\frac{\sinh \frac{1}{2}(\alpha-s i \gamma)}{\sinh \frac{1}{2}(\alpha+s i \gamma)}
$$

and

$$
\exp i \phi(\alpha)=\frac{\sinh \frac{1}{2}(\alpha+2 i \gamma)}{\sinh \frac{1}{2}(\alpha-2 i \gamma)}
$$

With these conventions the dependence on heterogeneities in the vertical spaces appears only in the Bethe equations. Eigenvalues depend on the horizontal parameters $u, s$ and the numbers $\alpha_{k}$ as in the homogeneous case. Complete expressions, which involve a sum of $s+1$ terms, can be found in [19]. The dominant term in the thermodynamic limit reads (with proper normalization)

$$
\Lambda_{N}^{s s^{\prime}}(u) \approx \prod_{k} \exp \left\{-i\left[p_{s^{\prime}}\left(\alpha_{k}+2 i u\right)-\pi\right]\right\}, N \rightarrow \infty
$$

For more details and references see for instance [19].

\section{2. $R S O S$ models}

If $q$ is a root of unity, $q=\exp (i \pi / t)$ where $t$ is an integer, we can also construct the Restricted Solid On Solid (RSOS) models corresponding to the quantum group $U_{q} s l(2)$. States in such a model are associated with faces of the lattice, and they take values $l_{j}=$ $1, \ldots, t-1$. The adjacency conditions between neighbouring faces depend on the spin associated with the edge between them [20][21]. The Boltzmann weights are associated with vertices of the lattice and they depend on the states of the neighbouring four faces. These weights are related to the vertex R-matrices via $6 \mathrm{j}$ calculus 222] 23.

Fix spins $s_{1}, \ldots, s_{N}$. The corresponding vertical space of states in the RSOS model, $\mathcal{H}_{s_{1}, \ldots, s_{N}}^{R S O S}$ with periodic boundary conditions, is best described by its basis

$$
\left\{l_{1}, \ldots, l_{N}\right\},\left(l_{i}-l_{i+1}+s_{i}\right) / 2 \in\left\{0, \ldots, s_{i}\right\}, s_{i}<l_{i}+l_{i+1}<2 t-s_{i}
$$

According to [21], 24] the eigenvectors of the RSOS transfer matrix are obtained from the Bethe equations

$$
\prod_{j=1}^{j=N} \exp \left[i p_{s_{j}}\left(\alpha_{k}-2 i u_{j}\right)\right] \prod_{l=1}^{l=M} \exp \left[i \phi\left(\alpha_{k}-\alpha_{l}\right)\right]=-\omega^{2}
$$


where we now have the constraint $M=\sum_{j} s_{j} / 2$ and $\omega^{t}=-(-)^{M} y(\operatorname{Im} \omega \neq 0)$ and $y$ is the eigenvalue of the operator given by

$$
Y_{l_{1}, \ldots, l_{N}}^{l_{1}^{\prime}, \ldots, l_{N}^{\prime}}=\prod_{i=1}^{i=N} \delta\left(l_{i}, t-l_{i}^{\prime}\right)
$$

that commutes with the RSOS transfer matrix. Eigenvalues of the RSOS transfer matrix are given by a sum of $s+1$ terms as in the vertex case, each term being however weighed by different powers of $\omega$. For complete expressions see [24] . These eigenvalues are a subset of the eigenvalues of the vertex model with $\omega=\exp \kappa(2.3)$ and in the spin $s^{z}=0$ sector [25].

\subsection{Arrays}

We shall consider further only two choices of parameters $s_{1}, \ldots, s_{N}$ and $u_{1}, \ldots, u_{N}$ :

Case I: we choose $s_{1}=\ldots=s_{N}=s$ and $-u_{2 j}=u_{2 j-1}=i \Lambda / 2$ for $j=1, \ldots, N / 2$.

Case II: we choose $s_{2 j-1}=s_{1}, s_{2 j}=s_{2}$ and $-u_{2 j}=u_{2 j-1}=i \Lambda / 2$ for $j=1, \ldots, N / 2$.

In both cases we assume $N$ to be even and we consider transfer matrices as generating functions for hamiltonians of quantum spin models (of generalized XXZ or RSOS type). For hamiltonians we choose

$$
H_{I}=-\left.\frac{1}{t} \frac{d}{d u} \ln \left[t^{s}(i \Lambda / 2+u)\left(t^{s}(-i \Lambda / 2-u)\right)^{-1}\right]\right|_{u=0}
$$

and

$$
H_{I I}=-\left.\frac{1}{t} \frac{d}{d u} \ln \left[t^{s_{1}}(i \Lambda / 2+u)\left(t^{s_{2}}(-i \Lambda / 2-u)\right)^{-1}\right]\right|_{u=0}
$$

so we have a total of four possible cases.

In all cases the models consist of an even and odd sublattice and hamiltonians commute with the total translation operator $\mathcal{T}: j \rightarrow j+2$. Hamiltonians are local and can be written

$$
H=H_{+}+H_{-}
$$

with

$$
H_{+}(\Lambda)=-\frac{1}{t}\left[\sum_{j \text { odd }} \dot{R}_{j+1, j}(i \Lambda)+\sum_{j \text { even }}\left(R_{j+2, j+1}(i \Lambda)\right)^{-1} \dot{R}_{j+2, j}(0) R_{j+2, j+1}(i \Lambda)\right]
$$


and

$$
H_{-}(\Lambda)=-\frac{1}{t}\left[\sum_{j \text { even }} \dot{R}_{j-1, j}(-i \Lambda)+\sum_{j \text { odd }} R_{j-2, j-1}(-i \Lambda) \dot{R}_{j-2, j}(0)\left(R_{j-2, j-1}(-i \Lambda)\right)^{-1}\right] \text {, }
$$

where dots stand for $\frac{d}{d u}$ and R-matrices implicitely carry spin upper indices in agreement with the arrays I and II, and correspond to XXZ type or RSOS models.

In terms of Bethe ansatz solutions eigenenergies of these hamiltonians read from (2.7)

$$
E_{I}=-\frac{2}{t} \sum_{k} \dot{p}_{s}\left(\alpha_{k}+\Lambda\right)+\dot{p}_{s}\left(\alpha_{k}-\Lambda\right)
$$

and

$$
E_{I I}=-\frac{2}{t} \sum_{k} \dot{p}_{s_{1}}\left(\alpha_{k}+\Lambda\right)+\dot{p}_{s_{2}}\left(\alpha_{k}-\Lambda\right)
$$

(this corresponds to antiferromagnetic interactions. For the ferromagnetic case switch the sign of $E$ ). The momentum of the corresponding state reads

$$
P_{I}=\sum_{k} p_{s}\left(\alpha_{k}+\Lambda\right)+p_{s}\left(\alpha_{k}-\Lambda\right)
$$

and

$$
P_{I I}=\sum_{k} p_{s_{1}}\left(\alpha_{k}+\Lambda\right)+p_{s_{2}}\left(\alpha_{k}-\Lambda\right)
$$

\subsection{Physical comments}

Consider first array I. Call $\Delta$ the spatial period of the system (equal to two lattice spacings in the original square lattice). Call $\tau$ the translation operator $j \rightarrow j+1$ so $\tau^{2}=\mathcal{T}$. Then $t^{s}(i \Lambda / 2) \tau$ and $\tau\left(t^{s}(-i \Lambda / 2)\right)^{-1}$ describe vertical propagation as in the following figure
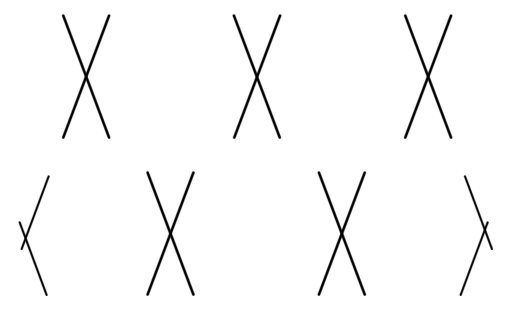

while $t^{s}(i \Lambda / 2)$ and $\left(t^{s}(-i \Lambda / 2)\right)^{-1}$ describe propagation along the right and left diagonal. The system can thus be intuitively considered as a "lattice light-cone" where the vertices represent interactions between $s+1$ "bare" particles (see [26] for a related approach). The natural hamiltonian associated with this description is given by 
$H_{I}=\frac{1}{i} \ln \left[t^{s}(i \Lambda / 2)\left(t^{s}(-i \Lambda / 2)\right)^{-1}\right]$. It is however non local, and this causes some difficulties (see later). Array II would have a similar interpretation but with the left (right) diagonal carrying spin $s_{1}\left(s_{2}\right)$. Note that in this case the only interactions are between left and right moving bare particles. Let us emphasize that in such a picture we are dealing with quantum mechanics at real time.

In the limit $\Lambda \rightarrow \infty$ the first term in (2.13) and (2.14) decays exponentially while the second one remains of order one. In that limit, the second term in (2.13) and (2.14) describes a RSOS hamiltonian with some special boundary conditions, coupling respectively only even (odd) sites. In the limit $\Lambda \rightarrow \infty$ the hamiltonian (2.12) is thus the sum of two decoupled RSOS hamiltonians. When $\Lambda$ is finite, they interact due to the first terms in (2.13) and (2.14). It is easy to see on the other hand that the transfer matrix $t^{s}(i \Lambda / 2)\left(t^{s}(-i \Lambda / 2)\right)^{-1}$ becomes trivial in the limit $\Lambda \rightarrow \infty$.

\section{Thermodynamic limit}

We now consider the thermodynamic limit $N \rightarrow \infty$. For the computation of thermodynamic quantitites, it is expected that the only relevant solutions of (2.4) are given in terms of strings [27]. For technical simplicity we will restrict in the following to values of the anisotropy parameter $q=\exp (i \gamma)$, where $\gamma=\pi / t, t$ being an integer.

\section{1. $X X Z$ type quantum chains}

For the vertex or generalized XXZ model case the solutions of (2.4) are made of $n$ strings of length $n=1, \ldots, t-1$ and of the $\left(n=1^{-}\right)$antistring of length 1 [27] (recall that an $n$ string is a set of $n$ complex numbers $\alpha_{k}^{(l)}=\alpha_{k}^{n}+i l \gamma$ with $l=n-1, n-3, \ldots,-n+1$ and an antistring of length one is a complex number with imaginary part equal to $\pi$ ). Regrouping the numbers $\alpha$ into their various strings leads to equations similar to (2.4) but for the (real) centers of the strings

$$
\prod_{j=1}^{j=N} \exp \left[i p_{n, s_{j}}\left(\alpha_{k}^{n}-2 i u_{j}\right)\right] \prod_{m l} \exp \left[i \phi_{n, m}\left(\alpha_{k}^{n}-\alpha_{l}^{m}\right)\right]=-1,
$$

where the second product is taken over all possible strings and then over each center. In the thermodynamic limit equation (3.1) gives the following integral equations for the densities $\rho_{n}, \tilde{\rho}_{n}$ of strings and holes

$$
\sum_{s_{j}} \dot{p}_{n, s_{j}} \star \mathcal{P}_{s_{j}}+\sum_{m} \dot{\phi}_{n, m} \star \rho_{m}=2 \pi \epsilon\left(\rho_{n}+\tilde{\rho}_{n}\right) .
$$


where $\mathcal{P}_{s}$ encodes the distribution of the heterogeneous spectral parameters for the spins $s$ (eg a sum of two delta functions or a single delta function in the array I or II) and $\epsilon=-1$ for the antistring.

In the following we set $a_{n, s} \equiv \dot{p}_{n, s}$ and $A_{n, m} \equiv 2 \pi \delta_{n, m} \delta-\dot{\phi}_{n, m}$. The various above functions are more conveniently expressed in terms of the Fourier transforms of their derivatives. For $n, s=1, \ldots, t-1$ one has

$$
\hat{a}_{n, s}=\frac{\sinh (n x) \sinh [(t-s) x]}{\sinh (t x) \sinh (x)}, n \leq s
$$

and

$$
\hat{A}_{n, m}=2 \frac{\cosh (x) \sinh (n x) \sinh [(t-m) x]}{\sinh (t x) \sinh (x)}, n \leq m .
$$

when one or two of the labels involve the antiparticle one has

$$
\hat{a}_{1^{-}, n}=-\frac{\sinh (s x)}{\sinh (t x)}
$$

and

$$
\begin{gathered}
\hat{A}_{1^{-}, n}=-2 \cosh (x) \frac{\sinh (n x)}{\sinh (t x)}, n \neq 1^{-}, n \neq t-1, \\
\hat{A}_{1^{-}, t-1}=-\frac{\sinh [(t-2) x]}{\sinh (t x)}, \hat{A}_{1^{-}, 1^{-}}=\hat{A}_{11}
\end{gathered}
$$

and the kernels are symmmetric otherwise.

Once the thermodynamic limit has been taken we compute the density of free energy of the infinite chain (with still finite lattice spacing) $\mathcal{F}=\mathcal{E}-T \mathcal{S}$, where $T$ is the temperature and $\mathcal{E}, \mathcal{S}$ are the densities of energy and entropy respectively. The formula for the entropy is the $N \rightarrow \infty$ limit of the combinatorial entropy of distribution of holes and particles [28]

$$
\mathcal{S}=\sum_{n} \int_{-\infty}^{\infty}\left[\left(\rho_{n}+\tilde{\rho}_{n}\right) \log \left(\rho_{n}+\tilde{\rho}_{n}\right)-\rho_{n} \log \left(\rho_{n}\right)-\tilde{\rho}_{n} \log \left(\tilde{\rho}_{n}\right)\right]
$$

The expression for the energy depends on the particular array. For array I one has

$$
\mathcal{E}_{I}=-\frac{2}{t} \sum_{n} \int_{-\infty}^{\infty}\left[a_{n, s}(\alpha+\Lambda)+a_{n, s}(\alpha-\Lambda)\right] \rho_{n}(\alpha) d \alpha
$$

and for array II

$$
\mathcal{E}_{I I}=-\frac{2}{t} \sum_{n} \int_{-\infty}^{\infty}\left[a_{n, s_{1}}(\alpha+\Lambda)+a_{n, s_{2}}(\alpha-\Lambda)\right] \rho_{n}(\alpha) d \alpha
$$




\section{2. $R S O S$ quantum chains}

We can follow the same steps for the RSOS chain. The additional phases in (2.9) seem to disappear in the equations for densities in the thermodynamic limit, but in fact as discussed in [24], they suppress the $t-1$ string and the antistring. This implies moreover that for the $t-2$ string there are no holes. One can then eliminate $\rho_{t-2}$ in the various equations to get a system exactly identical to (3.2) but where $n=1, \ldots, t-3$ and where $t$ is replaced by $t-2$ in the various kernels [24].

\subsection{Thermodynamic equilibrium for $X X Z$ type quantum chains}

The minimization of the free energy leads to coupled equations for the densities [27] [28] . For array I we have the following. Introducing $\epsilon_{n}=T \log \left(\tilde{\rho}_{n} / \rho_{n}\right)$ we get, after inversion of the matrix in (3.2),

$$
-e(\alpha) \delta_{n, s}=\epsilon_{n}-T s \star \sum_{m} N_{n, m}^{X X Z} \log \left[1+\exp \left(\epsilon_{m}\right) / T\right] .
$$

where we have defined

$$
e(\alpha) \equiv s(\alpha+\Lambda)+s(\alpha-\Lambda)
$$

and

$$
s(\alpha) \equiv \frac{1}{\cosh (t \alpha / 2)} .
$$

In the last expression $N_{n, m}^{X X Z}$ is the adjacency matrix of the following diagram

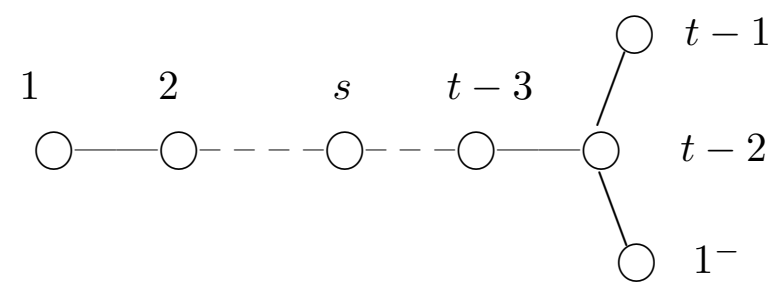

For the free energy per unit length we find

$$
F_{I}=-\int_{-\infty}^{\infty}\left\{T \log \left[1+\exp \left(\epsilon_{s} / T\right)\right]+a_{s, s}(\alpha)\right\} e(\alpha) d \alpha .
$$

Similarly for the array II, inversion of the matrix in (3.2) now leads to

$$
-e_{s_{1}}(\alpha) \delta_{n, s_{1}}-e_{s_{2}}(\alpha) \delta_{n, s_{2}}=\epsilon_{n}-T s \star \sum_{m} N_{n, m}^{X X Z} \log \left[1+\exp \left(\epsilon_{m} / T\right)\right],
$$

where

$$
e_{s_{1}}(\alpha)=s(\alpha+\Lambda), e_{s_{2}}(\alpha)=s(\alpha-\Lambda),
$$

and for the free energy

$F_{I I}=-\int\left\{T \log \left[1+\exp \left(\epsilon_{s_{1}} / T\right)\right]+a_{s_{1} s_{1}}\right\} e_{s_{1}}(\alpha) d \alpha+\left\{T \log \left[1+\exp \left(\epsilon_{s_{2}} / T\right)\right]+a_{s_{2} s_{2}}\right\} e_{s_{2}}(\alpha) d \alpha$ 


\subsection{Thermodynamic equilibrium for RSOS type quantum chains}

Things are very similar in that case. The only difference is that sums over solutions run only over $n=1, \ldots, t-3$ strings in (3.11), (3.15), (3.14) and (3.17) while in (3.11) and (3.15) $N_{n, m}^{R S O S}$ is the incidence matrix of the diagram

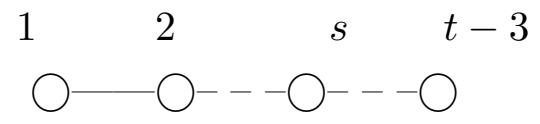

\section{$3.5 T \rightarrow 0$ limit for array $I$}

Consider first the ground state of array I at $T=0$ (in this paragraph the distinction between generalized XXZ and RSOS chains is simply encoded in the two possible incidence diagrams). System (3.11) reads then

$$
-e(\alpha) \delta_{n, s}=\epsilon_{n}-s \star \sum_{m} N_{n, m} \epsilon_{m}^{+},
$$

where $\epsilon^{+}(x)=\epsilon(x) H(\epsilon(x))$ is the positive part of the $\epsilon$ function ( $\mathrm{H}$ being the Heaviside step function). The solution of this system is $\epsilon_{n}^{+}=0$ and $\epsilon_{n}^{-}=-e \delta_{n s}$. From (3.18) we see that the ground state is filled with $s$ strings. Excitations above this ground state are made of holes in the sea of $s$ strings, with excitation energy precisely equal to $e$ (other strings are important in determining for instance the spin of the excitations, but they do not contribute to the energy and momentum). The momentum of the excitations is similarly found to be

$$
p=2 \tan ^{-1}\left(\frac{\sinh t \alpha / 2}{\cosh t \Lambda / 2}\right)+\pi .
$$

and as in [29] they always occur in pairs (with our convention $p$ is defined modulo $4 \pi$ ). There are two Brillouin zones. In the first one the model has massive excitations with a gap at $p=\pi$ that vanishes exponentially with $\Lambda$. In the second zone the model has massless excitations around $p=0,2 \pi$. This is true both for XXZ and RSOS chains provided $s \leq t-3$. In our subsequent discussion we discard the finite parts of the momenta. The role of these contributions in the scaling limit is similar to the one in spin $s=1$ case discussed eg in [30 


\section{5. $T \rightarrow 0$ limit for array II}

In that case the ground state is filled with $s_{1}$ and $s_{2}$ strings and the excitations above it are made of holes in the sea, with excitation energies equal to $e_{s_{1}}, e_{s_{2}}$. The momentum of excitations is similarly found to be

$$
p_{s_{1}}=2 \tan ^{-1}\{\exp [t(\alpha+\Lambda) / 2]\}, p_{s_{2}}=2 \tan ^{-1}\{\exp [t(\alpha-\Lambda) / 2]\} .
$$

There are massless excitations around $p=0, \pi$ in both cases. Again this is true for both XXZ and RSOS chains provided $s_{1}, s_{2} \leq t-3$.

\section{The scaling limit}

\subsection{XXZ type chain and array $I$}

In the first Brillouin zone the dispersion relation exhibits a gap that vanishes in the limit $\Lambda \rightarrow \infty$ as

$$
m \Delta \equiv \delta E \approx 4 \exp (-t \Lambda / 2),
$$

Let us consider the scaling limit. Let $N>>1$ and at the same time $\Delta<<1$ in such a way that the dimensional length of the chain $L=\Delta N$ is much bigger than the correlation length $\xi \equiv \frac{\Delta}{\delta E}$, the latter itself remaining finite. Hence we need

$$
\Lambda, N>>1, \Delta<<1, N \delta E>>1, \Delta \propto \delta E
$$

We then concentrate on the contributions of order $\Delta$ to the hamiltonian. At finite rapidity, that is very close to the origin of the first Brillouin zone, we then find for the excitation energies

$$
e(\alpha) \approx 4 e^{-t \Lambda / 2} \cosh (t \alpha / 2) \equiv \Delta m \cosh \theta,
$$

where the mass $m \equiv 1 / \xi$, and the physical rapidity $\theta \equiv t \alpha / 2$. Similarly for the momentum

$$
p(\alpha) \approx 4 e^{-t \Lambda / 2} \sinh (t \alpha / 2) \equiv \Delta m \sinh \theta,
$$

Relations (4.3) and (4.4) correspond to relativistic particles of mass $m$. Excitations at finite distance from the origin in the first Brillouin zone have finite energy and will disappear in the scaling limit. 
In the second Brillouin zone we also have excitations with energy of order $\Delta$. As $\alpha>>\Lambda$ ( $\Lambda$ being itself very large $)$ we have

$$
e_{L}(\alpha) \approx 2 e^{t \Lambda / 2} e^{-t \alpha / 2}
$$

with

$$
p_{L}(\alpha) \approx-e_{L}
$$

Similarly as $\alpha<<-\Lambda$ we have

$$
e_{R}(\alpha) \approx 2 e^{t \Lambda / 2} e^{t \alpha / 2}
$$

with

$$
p_{R}(\alpha) \approx e_{R}
$$

As in [29] there are only even number of the different types of excitations and the finite contributions to the momenta can be discarded. These relation correspond to massless left (respectively right) moving particles.

As $\Delta \rightarrow 0$ we expect the following

$$
H_{l a t t} \approx N \mathcal{E}_{0}+H_{\text {scal }} \Delta
$$

where $H_{\text {scal }}$ is the hamiltonian of the continuum field theory obtained in the scaling limit. On the other hand from [31], [32] we observe that the thermodynamic equations (3.11] of our system, for energies of order $\Delta$, coincide in the first Brillouin zone with the so called Thermodynamic Bethe Ansatz equations 2 for the spin $s$ or fractional supersymmetric sineGordon model [7] with coupling constant

$$
\frac{\beta_{S G}^{2}}{8 \pi}=\frac{\bar{t}}{s(s+\bar{t})},
$$

where $\bar{t}=t-s$ and $c_{U V}=\frac{3 s}{s+2}$, once the temperature in our problem has been identified as usual with the inverse radius $R$ in [31], [32]. Similarly in the second Brillouin zone the thermodynamic equations of our system for energies of order $\Delta$ coincide with the "massless TBA" equations for the generalized Gaussian model (a system made of a free boson and $Z_{s}$ parafermions) at radius of compactification appropriately related to (4.10) 33. The

2 Here by TBA we refer to thermodynamic analysis of a gas of relativistic particles in one dimension, with factorized scattering. 
excitations are then massless since one is describing a conformal field theory. Moreover, as $\Lambda>>1$, the regions of interest in the first and second Brillouin zone completely decouple since the kernel in the lattice equation (3.11) decays exponentially outside a region of finite size in $\alpha$ variable. We therefore conclude that

$$
H_{\text {scal }}=H_{S G-s}+H_{G-s}
$$

where the first term is the hamiltonian of the spin-s sine-Gordon model and the second term the hamiltonian of the generalized Gaussian model (a conformal invariant theory) on a cylinder of circumference $L$ with appropriate boundary conditions: $H_{G}=\frac{2 \pi}{L}\left(L_{0}+\bar{L}_{0}-\frac{1}{12}\right)$.

The identification of the scaling limit could be made completely by computing, from the lattice model [19] 34] [35], the $S$ matrix for the scattering of excitations. For doing so one interprets the holes in the sea as the physical particles. The other string solutions correspond then to pseudoparticles. In the present case three $\mathrm{S}$ matrices would have to be computed: the one describing the massive SG model and two others, identical ones, describing scattering between left (respectively right) "massless particles" in the conformal field theory. One would thus get three TBA diagrams like

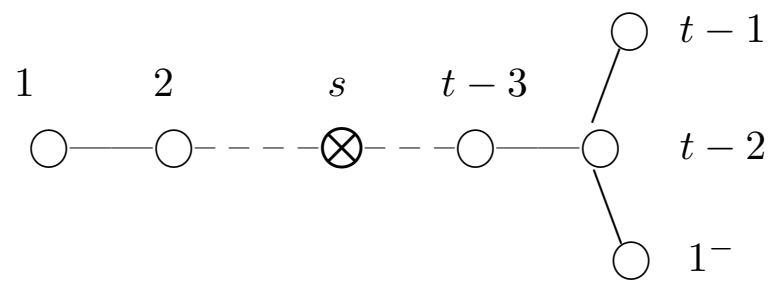

where the marked node corresponds to a massive particle of mass $m$, repsectively to a left or right moving particle. We comment more on massless scattering in section 5 where we also give a detailed example of an $\mathrm{S}$ matrix computation. Let us just mention that the $S$ matrices extracted in this fashion coincide with the known one for spin $s$ sine-gordon [7] and the ones for generalized Gaussian models [33] [29] [36].

To understand more directly what perturbed conformal field theory we are dealing with we finally consider [37] a limiting process similar to (4.2) but with the correlation length much bigger than $L$ ie

$$
\Lambda, N \rightarrow \infty, \Delta \rightarrow 0, N \delta E<<1, \Delta \propto \delta E
$$

In that limit we have

$$
H_{\text {scal }}=H_{\text {conf }}+\exp (-\Lambda) H_{\text {pert }},
$$


where $H_{\text {conf }}=H_{G-s}+H_{G-s}$ (this corresponds to (2.12) being a sum of two decoupled XXZ type models in the limit $\Lambda \rightarrow \infty)$ and $H_{\text {pert }}$ is determined by the first term in (2.13) and (2.14) . Comparing this with the formula giving the physical mass leads to the value of the conformal weight of the perturbation $h_{S G-s}=\frac{t-1}{t}$. This conformal weight could also be found for $s=1$ by noticing that taking the limit $\Lambda \rightarrow \infty$ the equations (2.4) with $s=1$ reduce to the ones of the Thirring model with bare mass $m_{0}=4 \sin \gamma \exp (-\Lambda)$ and bare rapidity $\alpha$ [34] [38]. Notice that the conformal weight does not depend on the spin $s$, ie it depends only on the skeleton of the TBA diagram and not on which node(s) are massive. This result can also be directly established at the level of the TBA equations [11].

\section{2. $R S O S$ chain and array $I$}

Things are quite similar in that case, with the additional truncation of (3.11). The thermodynamic equations (3.11) for energies of order $\Delta$ now coincide in the first Brillouin zone with the TBA equations for the theory $\mathcal{M A}_{s t}^{-}$written in [8]. Similarly in the second Brillouin zone the equations coincide with the massless TBA that describes minimal conformal field theories [33] . These three TBA are associated with the same diagram

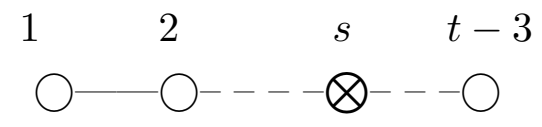

where the marked node corresponds to a massive particle of mass $m$, respectively a left or right moving particle. Equation (4.11) now reads

$$
H_{s c a l}=H_{\mathcal{M A}_{s t}^{-}}+H_{\mathcal{M}_{s t}},
$$

where the notations are explained in the introduction. In the limit (4.12) one finds

$$
H_{\text {scal }}=H_{\text {conf }}+\exp (-2 \Lambda) H_{\text {pert }},
$$

where $H_{\text {conf }}=H_{\mathcal{M}_{s t}}+H_{\mathcal{M}_{s t}}, H_{\text {pert }}$ is determined by the first term in (2.13) (2.14). The main difference with the unrestricted case discussed above is that the perturbation starts with order $\exp (-2 \Lambda)$ instead of order $\exp (-\Lambda)$ : this is due to the different spectral parameter dependence of the R-matrices (the different gradations from $\hat{U}_{q} s l(2)$ point of view [39] 3. As a consequence one finds $h=\frac{t-2}{t}$ as announced in the introduction.

To conclude this subsection we have established that array I provides a regularization of the interpolating theory $\mathcal{M} A_{s, t}^{-}$(plus a "background" $\mathcal{M}_{s t}$ theory that decouples).

3 The difference of exponent in (4.13) and (4.15) can also be observed in the ellpitic case: see the Boltzmann weights in [21]. 


\subsection{XXZ type chain and array II}

We consider again the scaling limit defined in (4.2). In the region $|\alpha|>>\Lambda$ (the second Brillouin zone) things are the same as for array I. They are quite different in the first Brillouin zone. In the present case we have, in the limit $\Lambda \rightarrow \infty$, and for finite rapidities,

$$
e_{s_{1}} \approx \frac{\Delta m}{2} e^{-\theta}, e_{s_{2}} \approx \frac{\Delta m}{2} e^{\theta},
$$

where $m, \theta$ are the same as before. It is straightforward to study as well the momentum of the excitations and one finds

$$
p_{s_{1}}=-e_{s_{1}}, p_{s_{2}}=e_{s_{2}},
$$

ie the dispersion relations for left and right moving massless particles. We thus have left

and right moving massless particles in both Brillouin zones. However, while in the second zone there is no left-right scattering since the two types of particles are widely separated in the rapidity variable $(\alpha>>\Lambda$ and $\alpha<<-\Lambda)$, in the first zone we expect to have non trivial left right scattering. This leads to a theory that is non scale invariant. In general however, one does not get a meaningful scaling limit due to the lack of symmetry between left and right sectors. The first step to recover this symmetry is to consider the RSOS version.

\subsection{RSOS chain and array II}

In the scaling limit, thermodynamic equations in the first Brillouin zone coincide with then with the TBA equations associated with a diagram of type $A_{n-3}$ with physical particles at nodes $s_{1}$ and $s_{2}$. For LR symmetry one then has to require $s_{1} \equiv s=t-2-s_{2}$, leading to the TBA diagram

$$
\begin{aligned}
& 1 \ldots \quad s \ldots \quad t-2-s \ldots t-3 \\
& \bigcirc---\bigotimes---\bigotimes--\bigcirc \\
& \frac{m}{2} e^{-\theta} \quad \frac{m}{2} e^{\theta}
\end{aligned}
$$

This TBA coincides then with the one conjectured in [8], [40] for the flow between successive minimal models. Hence we expect

$$
H_{\text {scal }}=H_{\mathcal{M A}_{s t}^{+}}+H_{\mathcal{M}_{s t}} .
$$


Observe on the other hand the following important symmetry for a RSOS model with $t-1$ heights (with appropriate normalization)

$$
R_{R S O S}^{s, s}(u)=R_{R S O S}^{s, t-2-s}\left[u+\frac{\pi}{2}\right] .
$$

(this can be derived from identities in [19] together with the observation that in the latter reference the spectral parameter $u$ in the weights $W^{p q}$ is shifted, when compared to ours, by the amount $\left.\frac{p-q}{2} \gamma\right)$.

Hence array II in the RSOS case is equivalent to array I, but with the cutoff $\Lambda$ acquiring an imaginary part $i \frac{\pi}{2}$. We can now make the identification with perturbed minimal models more complete by considering the limit (4.12). Due to the imaginary part of $\Lambda$ we observe indeed the change of sign of the coupling constant in (4.15) for the RSOS model:

$$
H_{s c a l}=H_{\mathcal{M}_{s t}}+H_{\mathcal{M}_{s t}}-\exp (-2 \Lambda) H_{\text {pert }}
$$

with $H_{\text {pert }}$ the same as in (4.13) . These results indicate that we have a regularization of the interpolating theory $\mathcal{M} A_{s, t}^{+}$. To complete the identification we shall compute the $S$ matrix in the following section.

Notice that, based on (4.19), it is tempting to consider also a type I array for the vertex model with a cutoff $\Lambda$ having imaginary part $\frac{i \pi}{2}$. From (4.13) it corresponds indeed to the spin $s$ sine-Gordon model with imaginary coupling constant. More detailed study of this model should be of interest [41].

\subsection{Computation of central charges}

In the framework of the TBA it is standard to compute the central charge in the UV and IR. Let us notice that it is also possible to extract these two numbers directly from the lattice model by considering appropriate limits. In the scaling limit we expect the entropy $\mathcal{S}(T, \Lambda)$ to become a function of the scaling variable $\frac{m}{\tau}$ where $\tau=\frac{T}{\Delta}$. The UV limit corresponds to $\tau>>m$ and the IR limit to $\tau<<m$. To extract the central charge from the lattice model one has to proceed as follows

$$
\mathcal{S}(T, \Lambda) \approx \frac{\pi T}{3} c_{I R} \text { if } T<<e^{-t \Lambda / 2}, T \rightarrow 0, \Lambda \rightarrow \infty
$$

and

$$
\mathcal{S}(T, \Lambda) \approx \frac{\pi T}{3} c_{U V} \text { if } T>>e^{-t \Lambda / 2}, T \rightarrow 0, \Lambda \rightarrow \infty
$$

For the RSOS model and array I the results of this computation are $c_{U V}=2\left(1-\frac{6}{t(t-1)}\right)$ and $c_{I R}=1-\frac{6}{t(t-1)}$ while for array II $c_{U V}$ is the same but $c_{I R}=1-\frac{6}{t(t-1)}+1-\frac{6}{(t-1)(t-2)}$. 


\section{The $S$ matrix for massless flows between minimal models}

\subsection{Massless $S$ matrices}

To start we briefly discuss the definition of massles $\mathrm{S}$ matrices. For integrable systems one can define scattering amplitudes via symmetry properties of eigenstates of the hamiltonian of the system under permutation of particles. We parametrize energy and momentum of left and right moving massless particles as in (4.16) and (4.17) so if $\Psi_{\theta^{R}, \theta^{L}}$ is an eigenstate of $H$ and $P$ one has

$$
H \Psi_{\theta^{R}, \theta^{L}}=\frac{m}{2}\left(\sum_{i} e^{\theta_{i}^{R}}+\sum_{j} e^{-\theta_{j}^{L}}\right) \Psi_{\theta^{R}, \theta^{L}},
$$

and

$$
P \Psi_{\theta^{R}, \theta^{L}}=\frac{m}{2}\left(\sum_{i} e^{\theta_{i}^{R}}-\sum_{j} e^{-\theta_{j}^{L}}\right) \Psi_{\theta^{R}, \theta^{L}},
$$

Consider a given set of rapidities $\theta_{1}^{R}, \ldots, \theta_{N}^{R}, \theta_{1}^{L}, \ldots, \theta_{M}^{L}$ and order them $\theta_{\sigma(1)} \leq \ldots \leq$ $\theta_{\sigma(N+M)}$ where $\sigma \in S_{N+M}$. To a given order characterized by some element $\tau$ of $S_{N+M}$ one can associate a linear space $\mathcal{H}_{\tau}\left(\theta^{R}, \theta^{L}\right)$ of dimension $C^{D(N+M)}$ where $D$ is the number of isotopic degrees of freedom. All these spaces are isomorphic and the isomorphism

$$
\mathcal{H}_{\tau}\left(\theta^{R}, \theta^{L}\right) \approx \mathcal{H}_{\tau^{\prime}}\left(\theta^{R}, \theta^{L}\right), \tau, \tau^{\prime} \in S_{N+M}
$$

defines some $\mathrm{S}$ matrix $S_{\tau, \tau^{\prime}}\left(\theta^{R}, \theta^{L}\right)$. The two particle $\mathrm{S}$ matrix is obtained in the case $\tau=\sigma$ and $\tau^{\prime}=\sigma \sigma_{i}$ where $\sigma_{i}$ transposes $i$ and $i+1$ as

$$
\begin{gathered}
S_{\sigma, \sigma_{i} \sigma}=S_{R R}\left(\theta_{j}^{R}-\theta_{k}^{R}\right) \text { if } \theta_{\sigma(i)}=\theta_{j}^{R}, \theta_{\sigma(i+1)}=\theta_{k}^{R}, \\
S_{\sigma, \sigma_{i} \sigma}=S_{L L}\left(\theta_{j}^{L}-\theta_{k}^{L}\right) \text { if } \theta_{\sigma(i)}=\theta_{j}^{L}, \theta_{\sigma(i+1)}=\theta_{k}^{L},
\end{gathered}
$$

and

$$
S_{\sigma, \sigma_{i} \sigma}=S_{L R}\left(\theta_{j}^{L}-\theta_{k}^{R}\right) \text { if } \theta_{\sigma(i)}=\theta_{j}^{L}, \theta_{\sigma(i+1)}=\theta_{k}^{R},
$$

with a similar equation for $S_{R L}$.

When we put the theory on a line of length $L$ this symmetry implies the form of the eigenfunctions

$$
\Psi_{\theta^{R}, \theta^{L}}\left(x_{1}, \ldots, x_{N+M}\right)=\sum_{\tau \in S_{N+M}} A_{\tau} \exp \left[i\left(p_{\sigma(1)} x_{1}+\ldots+p_{\sigma(N+M)} x_{N+M}\right)\right],
$$

where the amplitudes $A_{\tau}$ behave as (5.3) . The standard form of periodic boundary conditions [33] then follows. 


\section{2. $S$ matrix for $\mathcal{M} \mathcal{A}_{s t}^{+}$}

To get the $S$ matrix of the excitations we proceed as in [19] [35]. For simplicity we first consider the case $s=1$. Start from

$$
\frac{1}{2}\left[a_{n}^{(t-2)}(\alpha+\Lambda)+a_{n, t-3}^{(t-2)}(\alpha-\Lambda)\right]=2 \pi \tilde{\rho}_{n}+\sum_{m=1}^{t-3} A_{n, m}^{(t-2)} \star \rho_{m},
$$

where the subscript mean $t$ is replaced by $t-2$ in equations (3.3) up to (3.7) . Rename now $\sigma_{L} \equiv \tilde{\rho}_{1}, \sigma_{R} \equiv \tilde{\rho}_{t-3}$. The densities $\sigma_{L, R}$ now are considered as describing physical particles (they are densities of holes in the original Bethe ansatz due to the structure of the ground state and its excitations). Similarly rename $\sigma_{n} \equiv \rho_{n-1}, e_{L} \equiv e_{1}, e_{R} \equiv e_{t-3}$ and isolate the physical densities. After lengthy calculation one finds

$$
2 \pi \tilde{\sigma}_{n}+\sum_{m=1}^{m=t-5} A_{m, n}^{(t-4)} \star \sigma_{m}=a_{n}^{(t-4)} \star \sigma_{L}+a_{n, t-5}^{(t-4)} \star \sigma_{R}
$$

as well as

$$
2 \pi\left(\sigma_{L}+\tilde{\sigma}_{L}\right)=e_{L}+a_{1}^{(t-4)} \star \sigma_{L}+a_{t-5}^{(t-4)} \star \sigma_{R}-\sum_{n=1}^{n=t-5} a_{n}^{(t-4)} \star \sigma_{n},
$$

and

$$
2 \pi\left(\sigma_{R}+\tilde{\sigma}_{R}\right)=e_{R}+a_{t-5}^{(t-4)} \star \sigma_{L}+a_{1}^{(t-4)} \star \sigma_{R}-\sum_{n=1}^{n=t-5} a_{t-5, n}^{(t-4)} \star \sigma_{n},
$$

here $a, A$ refer to the same kernels as in (3.4) (3.7) but with $t$ replaced by $t-4$. As a consequence there is $L, R$ symmetry ie $A_{n, m}^{(t-4)}=A_{t-2-n, t-2-m}^{(t-4)}$. Taking now the limit $\Lambda \rightarrow \infty$, the equations (5.7) (5.8) (5.9) can be identified with the ones one would write for a theory with $\mathrm{L}$ particles of spin $1, \mathrm{R}$ particles of spin $t-3$ with factorized scattering given by $S_{L L}=S_{t-3}^{11}, S_{R R}=S_{t-3}^{t-5, t-5}=S_{L L}$ and $S_{L R}=S_{t-3}^{1, t-5}$ 目, where in each case $S$ is made of Boltzmann weights of the appropriate RSOS model with $t-3$ heights l $_{\text {and }}$ adjacency conditions depending on the spin, and the usual minimal prefactors ensuring unitarity and crossing symmetry 6. Indeed (5.7) just expresses the densities of pseudoparticles in the problem of computing the eigenvalues for passing a $L$ or $R$ particle through a set of $L$ and

4 These identifications hold up to a phase, which cannot be determined from the TBA equations

5 To be put in correspondence with the minima in the Landau Ginzburg picture

6 These identifications hold up to a phase, which cannot be determined from the TBA equations 
$\mathrm{R}$ particles with the densities $\sigma_{L}, \sigma_{R}$ (that is the eigenvalues of the "monodromy matrix" $T^{s}$ in section 2). Similarly (5.8) and (5.9) express the periodicity condition for the wave function of $\mathrm{L}$ and $\mathrm{R}$ particles.

Using the symmetry between spin 1 and spin $t-5$ in the RSOS version, one can

rewrite $S_{L R}(\theta)$ as $S^{11}\left[\theta+i \frac{(t-2) \pi}{2}\right]$ as well, in agreement with the result conjectured in [6].

The computation easily extends to the higher $s$ case. One finds then

$$
S_{L L}=S_{s+1}^{11} \otimes S_{t-1-2 s}^{11} \otimes 1, S_{R R}=1 \otimes S_{t-1-2 s}^{11} \otimes S_{s+1}^{11},
$$

and

$$
S_{L R}=1 \otimes S_{t-1-2 s}^{11}\left[\theta+i \frac{(t-2) \pi}{2}\right] \otimes 1,
$$

where $S_{n}$ is the S matrix based on the RSOS model with $n$ heights. In the above notation, left and right components of the tensor product correspond respectively to L and R RSOS models (with $s+1$ heights), while the middle component is as in the case $s=1$ above, ie it contains both L and R RSOS models (with $t-1-2 s$ heights), and the $\mathrm{S}$ matrix acts on LL, RR, LR respectively.

\subsection{Physical comments}

Notice that in the interacting theory we have non trivial LL,RR,LR scattering, while in the lattice light-cone picture only LR scattering occurs between the bare particles.

It may be useful to summarize the various correspondence between lattice RSOS models and thermodynamic equations, RSOS models in S matrices, and TBA. In all the previous sections the lattice model is based on quantum parameter $q=\exp (i \pi / t)$. This corresponds to a lattice RSOS model with $t-1$ heights. In the massive case, the continum limit is characterized by a quantum parameter $q_{\text {massive }}=\exp (i \pi /(t-1))$. The $\mathrm{S}$ matrix is based on a RSOS model with $t-2$ heights. The TBA diagram has $t-3$ heights $(t-4$ come from pseudoparticles, one is massive). In the massless case the continuum limit has parameter $q_{\text {massless }}=\exp (i \pi /(t-2))$. The $\mathrm{S}$ matrix is based on a RSOS model with $t-3$ heights. The TBA diagram has still $t-3$ heights $(t-5$ come from pseudoparticles, two massless). For instance it we take $t=5, s=1$ that is the lattice $A_{4}$ model, in the case of array I we get the massive flow from the tricritical Ising theory $(c=7 / 10)$ to the trivial fixed point, and in the case of array II the massless flow from tricritical Ising to Ising. 


\section{Generalizations}

The method can be extended easily to more complicated arrays, or to other algebras. As an example let us focus on $s o(2 r)$. As is generic in the higher rank case, the analysis of the Bethe ansatz equations, together with a string hypothesis for every color $a=1, \ldots, r$ leads to a TBA system that can be associated with a diagram in the plane order of roots versus color [37]. Restricting to the case $q=\exp (i \pi / 2 r)$ and turning moreover to the RSOS case there are only real solutions (1 strings) to take into account here, and the diagram is again a $D$ diagram, where this time the nodes are labelled by colors. We can then consider several kinds of arrays as before. We restrict now to ferromagnetic interactions 7. A simple case is the type I array built with the fundamental representation. Then one recovers the same results as in above (see discussion before eq (4.10) for $s=1$ ), due to the well known fact that the rational points of the sine Gordon model $\beta^{2}=8 \pi \frac{r}{r+1}$ can also be described as cosets $s o(2 r)_{1} \otimes s o(2 r)_{1} / s o(2 r)_{2}$ (a similar correspondence with the sine-Gordon model at $\beta^{2}=\frac{8 \pi}{r+1}$ would be observed in the antiferromagnetic case, where all nodes would be massive, corresponding to the bound states). This correspondence extends to higher spin. The most interesting case occurs with the spinor representations. Consider first the array of type I based say on positive chirality. One gets then in the limit $\Lambda \rightarrow \infty$, thermodynamic equations that coincide with the TBA associated with

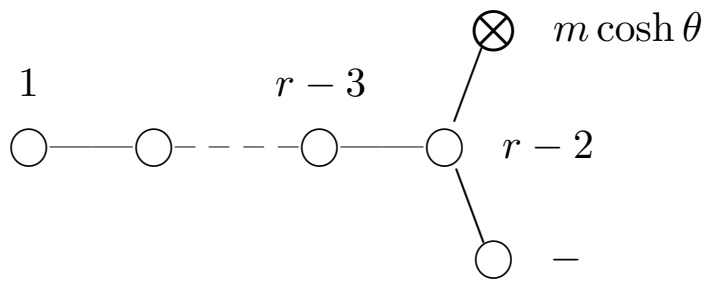

This corresponds to the massive perturbation of $Z_{r}$ parafermions by the operator of dimension $h=\frac{r-1}{r}$ 43]. For the array of type II, using the two chiralities for $s_{1}$ and $s_{2}$ one finds in the limit $\Lambda \rightarrow \infty$ thermodynamic equations that coincide with the TBA associated with

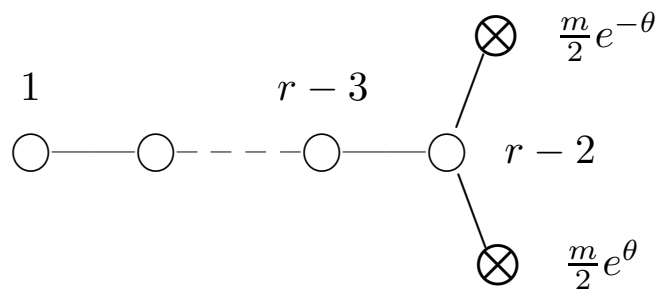

7 Recall that in level rank duality, ferro and antiferro interactions are exchanged [19] 42] 
which corresponds to the massless perturbation of parafermions with $c_{U V}=\frac{2(r-1)}{r+1}, c_{I R}=$ $1-\frac{6}{(r+1)(r+2)}$. If we now consider the $r \rightarrow \infty$ limit, these two possibilites provide integrable discretizations of the $O(3)$ non linear sigma model at $\Theta=0$ (resp. $\Theta=\pi$ ) following [43].

Finally, it is easy to read the $\mathrm{S}$ matrix for these two problems. For doing that we interpret the TBA diagram again as an $s u(2)$ diagram. In the first case the $A_{r-1}$ part then arises from the pseudoparticles and the additional leg indicates that the physical

particles have spin 2 . Hence $S=S_{r-1}^{22}$. In the second case we have to interpret the $A_{r-2}$ part as related to pseudoparticles, and the two additional legs correspond to the physical L and R particles. Then $S_{L L}=S_{R R}=S_{R L}=S_{r-2}^{11}$.

\section{Conclusion}

In conclusion we would like to notice that the approach where one chooses for hamiltonian (eg in the type I array)

$$
H_{I}=\frac{1}{i} \ln \left[t^{s}(i \Lambda / 2)\left(t^{s}(-i \Lambda / 2)\right)^{-1}\right]
$$

(as is done in [26] ) seems correct for the study of the scaling limit but breaks down in the perturbative analysis (4.12). In that case instead of equation (4.13) or (4.14) one gets a trivial unperturbed term, and the identification with perturbed conformal field theories becomes questionable. This problem is presumably due to the hamiltonian being non local.

We also would like to comment about the study of higher conserved quantities from the lattice. For simplicity we restrict to the massive case. Expand the energy (3.12) in the region $|\alpha|<\Lambda$, after renaming $e \rightarrow e^{(1)}$,

$$
e^{(1)}=4 \sum_{n=0}^{\infty} \exp \left[-(2 n+1) \frac{t \Lambda}{2}\right] \cosh \left[(2 n+1) \frac{t \alpha}{2}\right] \text {. }
$$

Instead of (2.10) we can consider other hamiltonians obtained by taking higher derivatives. Define therefore

$$
H_{I}^{(n)}=\left.i\left(\frac{i}{t}\right)^{n} \frac{d^{n}}{d u^{n}} \ln \left[t^{s}(i \Lambda / 2+u)\left(t^{s}(-i \Lambda / 2-u)\right)^{-1}\right]\right|_{u=0}
$$

Its eignvalues are given by

$$
E_{I}^{(n)}=\left(-\frac{2}{t}\right)^{n} \sum_{k} p_{s}^{(n)}\left(\alpha_{k}+\Lambda\right)-(-1)^{n} p_{s}^{(n)}\left(\alpha_{k}-\Lambda\right) .
$$


The analysis of thermodynamics and excitations is very similar to the case $n=1$. One finds the energies of excitations to be given by

$$
e^{(n)}(\alpha)=\left(-\frac{2}{t}\right)^{n-1}\left(\frac{d}{d \Lambda}\right)^{n-1} e^{(1)}(\alpha)
$$

Expand now these quantities as (7.1) . One finds for instance

$$
e^{(2)}=4 \sum_{n=0}^{\infty}(2 n+1) \exp \left[-(2 n+1) \frac{t \Lambda}{2}\right] \cosh \left[(2 n+1) \frac{t \alpha}{2}\right] \text {. }
$$

All these quantities have dominant behaviour $e^{(n)}(\alpha) \approx 4 e^{-t \Lambda / 2} \cosh (t \alpha / 2)$. However by forming linear combinations of them we can extract higher order terms. For instance

$$
e^{(2)}-e^{(1)} \approx 8 e^{-3 t \Lambda / 2} \cosh (3 t \alpha / 2)
$$

Hence

$$
H_{\text {latt }}^{(2)}-H_{\text {latt }}^{(1)} \approx N \mathcal{E}_{0}+\Delta^{3} H_{\text {scal }}^{(3)}
$$

with obvious generalizations to higher orders. In the same way as $H_{\text {scal }}^{(1)}$ is the quantum sine-Gordon hamiltonian, the $H_{\text {scal }}^{(2 n+1)}$ are the higher hamiltonians - non trivial for odd grade only - of the integrable hierarchy [44 [9]. They commute since the various latttice hamiltonians (7.2) used in their construction do.

Acknowledgments: N.Yu Reshetikhin was supported by NSF under grant No. DMS9015821 and the Sloane foundation. H.Saleur by DOE under grant No. DE-FG0384ER40168 and the Packard foundation. 


\section{References}

[1] A.B.Zamolodchikov, Int. Jour. Mod. Phys. A3 (1988), 746.

[2] J.Cardy, A.Ludwig, Nucl. Phys. B287 (1987) 687.

[3] A.B.Zamolodchikov, Sov. J. Nucl. Phys. B287 (1987) 687.

[4] V.Dotsenko, V.Fateev, Nucl. Phys. B251 (1985) 691; G.Felder, Nucl. Phys. B317 (1989) 215.

[5] N.Yu Reshetikhin, F.Smirnov, Comm. math. Phys. 131 (1990) 157.

[6] P.Fendley, H.Saleur, Al.B.Zamolodchikov, "Massless flows between minimal models", preprints USC-93/003 and 93/004.

[7] D.Bernard, A.Leclair, Phys. Lett. B247 (1990) 309.

[8] Al.B.Zamolodchikov, Nucl. Phys. B366 (1991) 122.

[9] T.Eguchi, S.K.Yang, Phys. Lett. B224 (1989) 373.

[10] C.Ahn, D.Bernard, A.Leclair, Nucl. Phys. B346 (1990) 409.

[11] Al.B.Zamolodchikov, Nucl. Phys. B358 (1991) 524.

[12] L.D.Faddeev, N.Yu Reshetikhin, Ann. Phys. 167 (1986) 227.

[13] A.G.Izergin, V.E.Korepin, Lett. Math. Phys. 5 (1981) 199.

[14] L.D.Faddeev, A.Yu Volkov, Theor. Math. Phys. 92 (1992) 207.

[15] M.Luscher, Nucl. Phys. B117 (1976) 475.

[16] A.A.Belavin, V.G.Drinfeld, Soviet Scientific Reviews C4 (1984) 93.

[17] P.Kulish, N. Reshetikhin, E.Sklyanin, Lett. Math. Phys. 5 (1981) 393.

[18] M.Jimbo, Lett. Math. Phys. 10 (1985) 163.

[19] A.N.Kirillov, N.Yu Reshetikhin, J.Phys. A 20 (1987) 1565, 1587.

[20] M.Jimbo, T.Miwa, M.Okado, Nucl. Phys. B300 (1988) 74.

[21] G.E.Andrews, R.J.Baxter, P.J.Forrester, J.Stat. Phys. 35 (1984) 193.

[22] V.Pasquier, Comm. Math. Phys. 118 (1988) 355.

[23] A.N.Kirillov, N.Yu Reshetikhin, in "Infinite-dimensional Lie algebras and groups", ed. V.G.Kac, World Scientific, Singapore (1989).

[24] V.V.Bazhanov, N.Yu Reshetikhin, J.Mod. Phys. A4 (1989) 115.

[25] V.Pasquier, H.Saleur, Nucl. Phys. B330 (1990) 523.

[26] C.Destri, H. de Vega, J.Phys. A22 (1989) 1329.

[27] M.Takahashi, M.Suzuki, Prog. Theor. Phys. 48 (1972) 2187.

[28] C.N.Yang, C.P.Yang, Phys. Rev. 147 (1966) 303.

[29] L.D.Faddeev, L.A.Takhtajan, Phys. Lett. 85A (1981) 375.

[30] I.Affleck, in "Fields, Strings and Critical Phenomena", Les Houches Lectures, E.Brezin et al. Editors, North Holland (1986).

[31] Al.B.Zamolodchikov, Phys. Lett. B253 (1991) 391.

[32] P.Fendley, H.Saleur, Nucl. Phys. B388 (1992) 609.

[33] P.Fendley, H.Saleur, "Lectures on massless flows", preprint USC -93-022. 
[34] V.Korepin, Th. Mat. Phys. 41 (1979) 169.

[35] A.Tsvelick, P.Wiegmann, Adv. in Physics 32 (1983) 453.

[36] A.B.Zamolodchikov, Al.B.Zamolodchikov, "Massless factorized scattering and sigma models with topological terms, preprint ENS-LPS-335-91.

[37] V.V.Bazhanov, N.Yu Reshetikhin, Prog. Th. Phys. 102 (1990) 301.

[38] H.Bergknoff, H.B.Thacker, Phys. Rev. D19 (1979) 3666.

[39] G.Felder, A.Leclair, in "Infinite Analysis", A.Tsuchiya et al. Eds., World Scientific, Singapore (1991).

[40] Al.B.Zamolodchikov, Nucl. Phys. B358 (1991) 497.

[41] S.Skorik, H.Saleur, in preparation.

[42] D.Altschuler, H.Saleur, Nucl. Phys. B354 (1991) 579.

[43] V.A.Fateev, Al.B.Zamolodchikov, Phys. Lett. B271 (1991) 91.

[44] R.Sasaki, L.Yamanaka, Adv. Studies in Pure Math. 16 (1988) 271. 